\title{
Searches for Gauge Mediated Supersymmetry at the Tevatron
}

\author{
Pierre Lutz ${ }^{* \dagger}$ \\ CEA Saclay \\ E-mail: lutz@in2p3.fr
}

\begin{abstract}
We report the results of searches for new physics in events with two photons and large missing transverse energy collected with both detectors at the Fermilab Tevatron collider. Several models of physics beyond the Standard Model motivate searches in this final state, in particular supersymmetry (SUSY) with gauge-mediated supersymmetry breaking. The D $\varnothing$ collaboration interprets also its search in the framework of universal extra dimensions (UED) with gravity-mediated Kaluza-Klein excitation decays. The results presented use $2.6 \mathrm{fb}^{-1}$ (CDF) and $6.3 \mathrm{fb}^{-1}$ (DØ) of data collected at the Fermilab Tevatron collider.
\end{abstract}

35th International Conference of High Energy Physics - ICHEP2010,

July 22-28, 2010

Paris France

\footnotetext{
* Speaker.

${ }^{\dagger}$ on behalf of the CDF and DØ Collaborations
} 


\section{Introduction}

Such a title is probably a bit misleading for the reader interested in a general search within the Gauge-Mediated Supersymmetry, since the only contributions (one from each Tevatron collaborations) we have to report on [1] [2] are indeed searches for new physics in diphoton events with large missing transverse energy, interpreted in the framew ork of such a model.

Gauge-Mediated Supersymmetry is a popular and attractive model, mostly because it drastically reduces the number of parameters from more than 100 to only 5 in its minimal implementation. In these models, Supersymmetry (SUSY) breaking occurs well below the Planck scale in a hidden sector of fields that are singlets under Standard Model (SM) gauge transformations. SUSY breaking is communicated to the visible sector by messengers that participate in both SM interactions and the dynamics of the hidden sector. SM superpartner masses are generated radiatively and are proportional to the effective SUSY breaking scale $\Lambda$. The gravitino $(\tilde{G})$ mass arises from the Super-Higgs mechanism and is much smaller than the masses of SM superpartners. The phenomenology of such models is then characterised by the decay of the next-to-lightest supersymmetric particle (NLSP) to its SM partner and a light gravitino. The important parameter in determining this NLSP is the number of messengers $N_{\text {mes }}$ which is chosen here equal to 1 , insuring that the NLSP is the lightest neutralino $\left(\chi_{1}^{0}\right)$. Assuming $R$-parity conservation, the primary process at the Tevatron is chargino and neutralino pair production, with a smaller contribution from slepton pairs. The produced superpartners initiate separate decay chains to the next-to-lightest SUSY particle $\chi_{1}^{0}$, and its decay is assumed to occur promptly with the dominant branching fraction yielding a photon and an essentially massless gravitino. The dominant feature of this model is thus $\chi_{1}^{0} \chi_{1}^{0} \rightarrow \gamma \tilde{G} \gamma \tilde{G}$ with additional activity (leptons and/or jets) motivating the search in the $(\gamma \gamma+$ missing transverse energy + anything) final state.

\section{Event selection summary}

Since both contributions are searches for the same final state, the event selection is rather similar: selection of di-photon events, finding the event vertex, computation of the transverse missing energy. We emphasize here only the improvements brought since their last publications on the subject [3] [4].

\subsection{Photon identification}

The D $\varnothing$ collaboration achieved an improved photon identification by using a neural network [5], with five input variables, describing the shape and isolation of the shower, allowing a good discrimination between true photons and misidentified jets, as can be seen on Fig. 1.

This technique, used also in other $\mathrm{D} \emptyset$ analyses, gives a factor around 2 improvement in rejection for same efficiency when compared with the previous photon identification tool, allowing an improved formulation of the background model.

\subsection{Vertexing}

There are on average several interactions per beam crossing. It is therefore important to correctly identify the vertex which both photons are coming from, since a wrong vertex would imply 

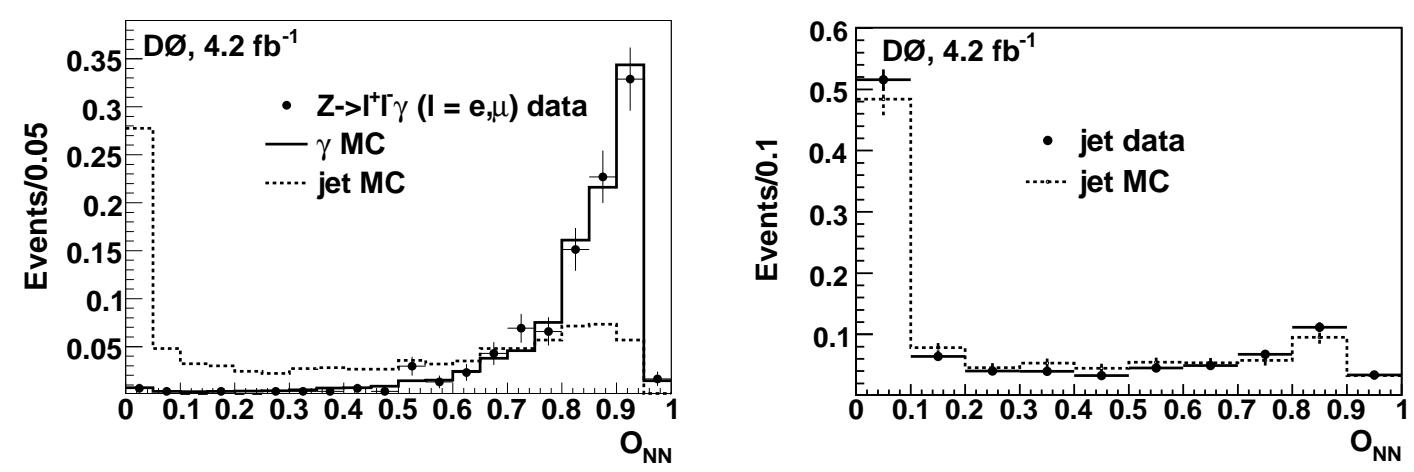

Figure 1: D $\varnothing$ : Comparison of the normalized output of the neural network $\mathrm{O}_{N N}$ spectra for photons and jets misidentified as photons in data and Monte-Carlo

a wrong transverse missing energy ! CDF uses their photon timing system to reject most of the cosmics induced di-photon and beam-halo events, while D $\emptyset$ makes use of their preshower to select events when both photons are pointing near a candidate primary vertex. In both cases, the consequence is that only prompt (or nearly prompt) decays of the neutralino NLSP can be considered for these analyses.

\subsection{Backgrounds and computation of the missing transverse energy}

There are three major sources of background for di-photon events with missing transverse energy: QCD events with fake missing transverse energy (MET), electroweak events with real MET, and various non-collision events where one (or more) of the photons and/or MET are not related to the collision. The first category, due to the huge QCD cross-sections, is by far the dominant background, even if the energy measurement fluctuations in the calorimeter (which lead to high values of fake MET) happen only in a small fraction of cases.

CDF developped an original method [6] to deal with such events, namely by selecting events based on a new variable, MetSig, which is the significance computed from the p-value to see fluctuations in the energy measurement to produce a MET greater or equal to the observed one. After estimating the MetSig distributions for all backgrounds, the expected MetSig distribution for the presample is shown in Figure 2(left).

$\mathrm{D} \emptyset$ used a more classical approach, modeling the MET shape with $\mathrm{Z} \rightarrow \gamma \gamma$ data sample (cross checked with SM diphoton Monte-Carlo simulation sample) for the SM part, and with misidentified jet data sample (at least one "photon" failing the Neural Net specification), then normalizing on MET $<10 \mathrm{GeV}$ by fitting the relative contributions of both SM diphoton and misID jet parts. The electron misID contribution is derived from the e $\gamma$ sample, and the whole background estimation was developed with the diphoton sample data blinded in the MET $>50 \mathrm{GeV}$ region.

\section{Results}

\subsection{CDF Collaboration}

Using $2.6 \mathrm{fb}^{-1}$ of data, there is no evidence for new physics and the data is well modeled by backgrounds alone as can be seen in Figure 2(right) With 0 data events and 1.2 expected, the 

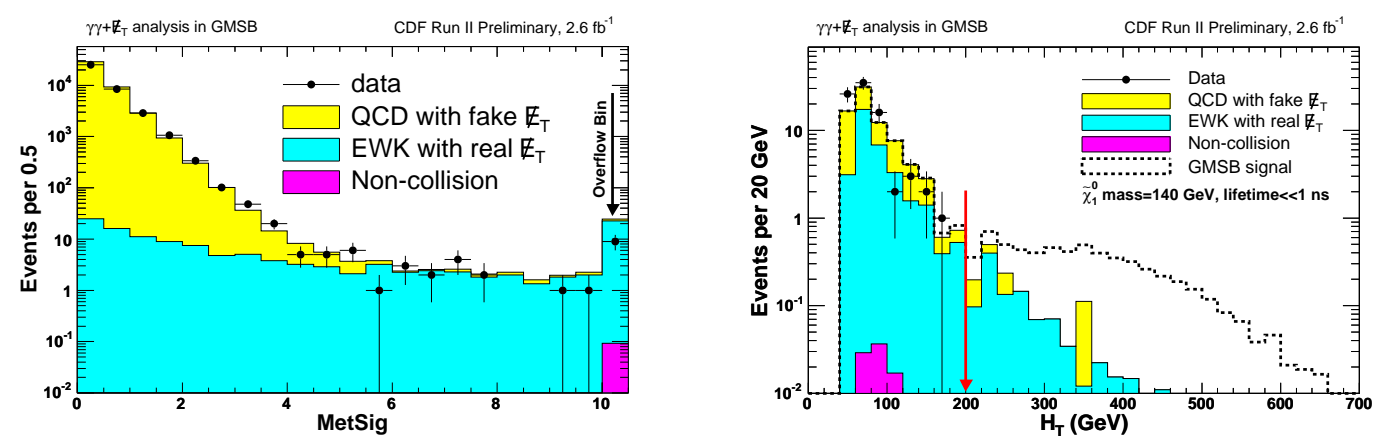

Figure 2: CDF : The background predictions for the presample (left).The various backgrounds together with the data just before the last cut (right).

collaboration sets limits on GMSB models with a $\chi_{1}^{0}$ mass reach of $149 \mathrm{GeV} / c^{2}$ for a $0 \mathrm{~ns}$ lifetime.

\subsection{DØ Collaboration}

No evidence for BSM physics is observed in the $\gamma \gamma$ sample extracted from $6.3 \mathrm{fb}^{-1}$ of data. Limits on the benchmark model is derived using a Poisson log-likelihood ratio test [7] incorporating the full missing transverse energy distribution. Figure 3 shows the observed MET distribution, consistent with SM (left) and the predicted cross section for the benchmark GMSB model, together with the 95\% C.L. expected and observed exclusion limits (right). Values of the effective SUSY breaking scale $\Lambda<124 \mathrm{TeV}$ are excluded at 95\% C.L. This translates into a limit on the mass of the lightest neutralino of $175 \mathrm{GeV} / c^{2}$ for this model.
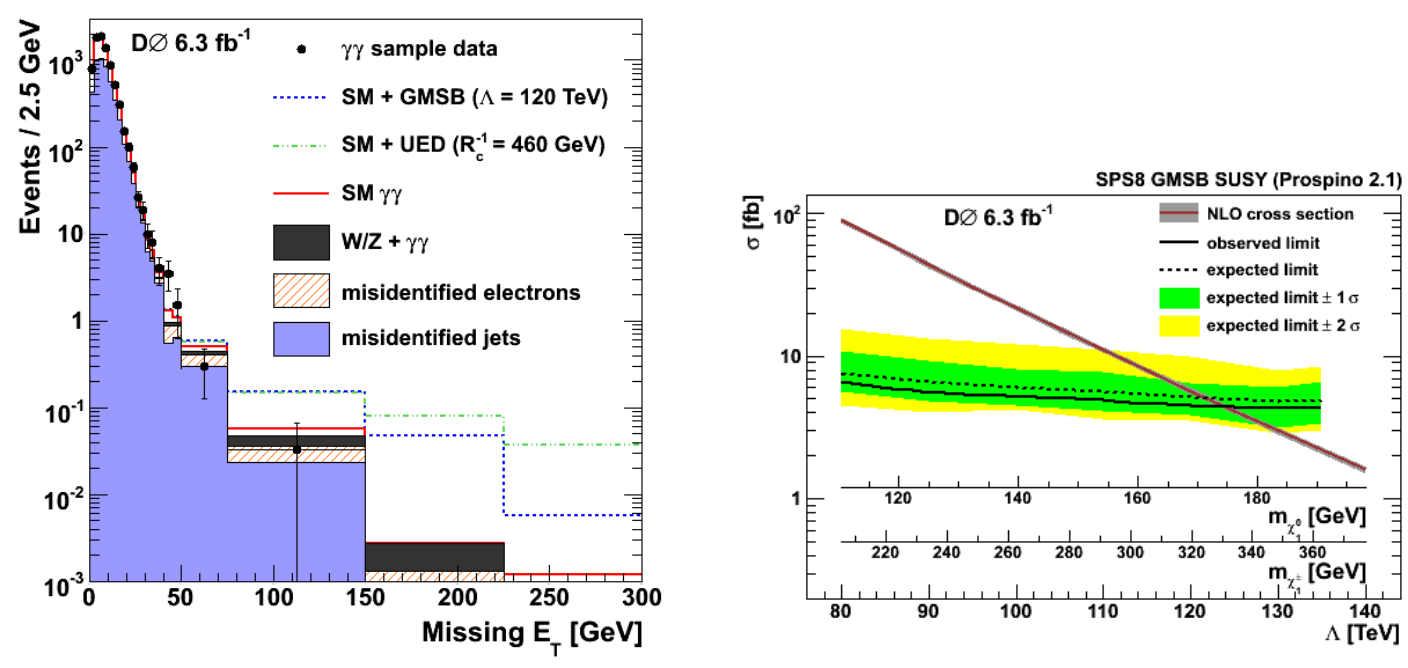

Figure 3: DØ: The observed missing transverse energy distribution (left), and the derived limits(right).

\section{Aknowledgements}

The speaker would like to thank the convenors of DØ New Physics group, Arnaud Duperrin and Gustaaf Broojmans, and the convenors of CDF exotics group, Oscar Gonzales and Tom Wright, for their help in preparing the presentation. 


\section{References}

[1] T. Aaltonen et al. (CDF Collaboration), Phys. Rev. Lett. 104 (2010) 011801.

[2] V.M. Abazov et al. (DØ Collaboration), Phys. Rev. Lett. 105 (2010) 251801.

[3] D. Acosta et al. (CDF Collaboration), Phys. Rev. D 71 (2005) 031104.

[4] V.M. Abazov et al. (DØ Collaboration), Phys. Lett. B 659 (2008) 856.

[5] V.M. Abazov et al. (DØ Collaboration), Phys. Lett. B 690 (2010) 108.

[6] R. Culbertson, A. Pronko, S. Yu, M. Goncharov, CDF public note 9339.

[7] T. Junk, Nucl. Inst. Meth. A 434 (1999) 435, and W. Fisher, FERMILAB-TM-2386-E (2006) 